Proceedings

The Conference met in morning and afternoon sessions on three consecutive days, September $3-5$, 1953.

FIRST SESSION

Topic: Photographic Catalogues. Chairman: D. Brouwer.

Papers were presented by O. Heckmann, R. H. Stoy and S. Vasilevskis.

SECOND SESSION

Topic: Meridian Circle Programs. Chairman: R. H. Stoy.

Papers were presented by F. P. Scott, Sir Harold Spencer Jones and W. S. McClenahan.

THIRD SESSION

Topic: Proper Motions, Parallaxes and Double Stars. Chairman: H. L. Alden.

Papers were presented by A. N. Vyssotsky, J. Schilt, D. L. Harris and K. Aa. Strand.

FOURTH SESSION

Topic: Time Determination; Instrumentation. Chairman: A. Danjon.

Papers were presented by A. Danjon, W. Markowitz, J. G. Baker, and W. J. Eckert.

FIFTH SESSION

Topic: Astrometric Applications. Chairman: O. Heckmann.

A paper was presented by W. W. Morgan.

SIXTH SESSION

Topic: Discussion of Resolutions. Chairman: G. M. Clemence.

The resolutions were presented by Sir Harold SPEnCER Jones.

\section{FIRST SESSION}

Sept. 3, 9:30 A.M. D. Brouwer, presiding

Following a welcome by STRAND and initial remarks by CLEMENCE, the meeting was turned over to BRouwer as Chairman of the first session. He pointed out that it was difficult to subdivide the subjects of astrometry to be discussed in the morning and afternoon sessions and suggested that the Conference therefore consider the subjects of star catalogues and meridian circle observations together. He mentioned the promise in automatic measuring and computing devices and announced that Yale has completed the catalogues for the stars between $+30^{\circ}$ and $-30^{\circ}$ declination and noted that the Cape Observatory was in the process of finishing its catalogues for the zones south of $-30^{\circ}$. He further called attention to the work of the Russians, of the Hamburg Observatory, and of the Lick Observatory, in connection with the use of external galaxies for a frame of reference. He pointed out the timeliness of the Conference on Astrometry for future work on positional catalogues.
Heckmann was then called on to present the first report. He gave a short summary of the contents of his paper, and called attention to the close relation of his own paper on a third AG Catalogue to those of VAsilevskis, Scott, and Vyssotsky. He asserted that large programs such as the formation of a third AG Catalogue would involve the solution of several separate problems and that the results would have important applications. The fundamental system should be connected to the extragalactic nebulae, thereby obtaining absolute proper motions in low galactic latitudes as well as in other parts of the sky. He spoke of the value of relating the proposed work to the Carte du Ciel and stated that in fact the idea of such a catalogue arose after re-reducing sample results from the Carte du Ciel. He cited as a concrete example of the usefulness of this procedure some recent work on a cluster in the neighborhood of $\alpha$ Persei. It had been discovered by EDDINGTON who with others failed to find its main sequence members and found only the early giants. Attempts to segregate main sequence cluster stars from the faint stars of the field were 
made by applying corrections for proper motions as determined from the AGKI and $\mathrm{AGK}_{2}$ and reducing to the epoch of the Carte du Ciel. The failure of this attempt led to new observations between 1930 and 1950 . From these and remeasurement of $\mathrm{AGK} 2$ plates new results have been obtained which are serving to identify the main sequence cluster members. He raised the question of a choice of reference stars for the third epoch and remarked on the advantage of taking the same stars as were used for the $\mathrm{AGK}_{2}$ in order to reduce the work of obtaining least-squares solutions. In addition results could then be published in about three volumes instead of the $\mathbf{I} 6$ volumes required for the AGK2. He suggested that exposures be made of sufficient length to record all stars of the Carte du Ciel, stipulating that though not all would be measured, they would be available for subsequent measurement.

Herget recalled that Schlesinger and Miss BARNEY have shown that the probable errors of places derived from the Astrographic Catalogue can be reduced by a factor of about 2, if new plate constants are computed with improved positions for the comparison stars. He asked what role was envisaged for the Astrographic Catalogues.

HeCKMANN replied that after the AGK2$\mathrm{AGK}_{3}$ system of positions and proper motions has been established, all the plate constants of the Astrographic Catalogue should be recomputed with comparison star places on this system.

Schilt expressed apprehension that longer exposure might result in a lower accuracy.

HECKMANN thought this would not be the case since the necessary increase in exposure time would be slight, and a grating could be used.

Vyssotsky inquired if the positions of the reference stars were to be obtained with meridian circles.

HeCKMANN explained that the program was to proceed in two steps. First, an attempt would be made to improve the fundamental system of the $\mathrm{FK}_{3}$; second, some $\mathrm{I}_{5}, 000$ stars of the northern hemisphere would be observed with meridian circles.

Vyssotsky stressed the need for absolute positions.

Heckmann asserted that this made it necessary to have a sufficient number of meridian circles take part in the project. He noted the unavailability of some of the instruments used for the AGK2, such as Breslau, Berlin-Babelsberg in eastern Germany, Bonn which has been dis- mantled, as well as the doubtful status of Heidelberg and Munich. Thus of the original six German observatories participating in the AGK2 program, only Hamburg can be certainly committed to an $A_{G K}$. Inasmuch as at least six meridian circles are desirable, this is one of the principal obstacles to the establishment of an $\mathrm{AGK}_{3}$.

SPEnCER Jones remarked that if the reference stars of the AGK2 were to be used again, many faint stars would be included, and that perhaps it would be better to restrict the program to brighter stars. To the suggestion that observations of faint stars might be made by using meridian circles equipped with two parallel wires, Spencer Jones suggested there might be a systematic difference between observations made with one wire and those made with two. He noted in addition that if observations were made fundamental, additional controls would be had on the $\mathrm{FK}_{3}$ system.

STOY inquired whether all reference stars of the AGK2 had been observed with all of the six meridian circles on the original program.

Heckmann explained that only Hamburg and Berlin-Babelsberg had observed the entire list of 14,000 stars and that the remaining observatories had each observed approximately 2,000 stars in overlapping areas.

BRouwer asked if it was HECKMANN's suggestion to obtain proper motions from $\mathrm{AGK}_{2}$ and $\mathrm{AGK}_{3}$ alone, without reference to other catalogues such as AGKI and the Yale zones.

HECKMANN agreed that after the systematic corrections to these catalogues had been determined with the aid of $\mathrm{AGK}_{2}$ and $\mathrm{AGK}_{3}$, they could also be used.

Stoy was then called upon to present his paper. He called attention to the fact that one instrument in the southern hemisphere was responsible for approximately a quarter of the sky. He therefore asserted that the limited resources must be made use of with maximum efficiency, and asked for suggestions as to how this might be done. He then gave a summary of past and current work on Cape catalogues. The procedure was outlined as follows: I. A selection of 1.25 comparison stars per square degree is made, principally from the Cordoba Durchmusterung. 2. Places are reduced to 1950.3 . Measures are made with the transit circle. 4. Standard coordinates are obtained. 5. Plates are measured. 6. Plate solutions are cal- 
culated. 7. Plate corrections are applied. 8. Proper motions are obtained.

In answer to a question by ClemenCe, Stoy noted that the work should be complete to the south pole by approximately i960, the $-30^{\circ}$ to $-56^{\circ}$ zone by next year, others in succeeding years.

BROUWER asked if failure to employ a grating would not lead to systematic differences, to which STOY replied that the magnitude equation is determined and applied. Brouwer questioned if this would not lead to a difference in accuracy for bright and faint stars.

Miss BARNEY stated her opinion that a grating is indispensable for the measurement of positions of brighter stars, which can otherwise not be measured as accurately as the fainter stars. She noted that measures in right ascension were easier to make than those in declination because of the elongation of first-order images in declination, the grating images having the same right ascension.

STRAND asked if it would not be better to use horizontal grating images because in the vertical grating images the effect of atmospheric dispersion would act in the same direction as the grating dispersion for one image and opposite for the other, making the two images very dissimilar.

BROUWER remarked that the horizontal images would become oblique through the combined effects of grating and atmospheric dispersion.

STRAND remarked that if these effects showed up due to using too wide a spectrum range, it would be better not to use gratings at all unless the spectrum range was cut down by use of filters. He also mentioned that the lack of proper magnitude compensation by Fox in the Dearborn parallax program has made these parallaxes practically useless, due to their large systematic errors.

STOY explained that magnitude corrections are made according to image size.

Clemence cautioned that magnitude differences may exist between the first and second programs if gratings are introduced, and that this may affect the proper motions.

MARKOWITZ asked if the magnitude error varies with image size, and if this same error would not be repeated on second plates.

STRAND called attention to the fact that quite generally one finds an increase of systematic errors with increase in magnitude differences, but that these errors usually vary from plate to plate both in regard to size and direction.
Miss Barney remarked that the measurements of large images cannot be made as accurately as those of small images; also that the Yale plates were measured in the direct and reversed positions.

Schilt stated that there would be a systematic difference between the guiding errors of bright and faint stars and that accidental errors would be a function of the brightness of the star.

HeCKMANn elaborated that the sources of error might be ascribed to guiding, prismatic effect and chromatic effect, of which the latter two can be eliminated only by a grating.

VASILEVSKIS noted that chromatic error might affect the mean of the positions of the two grating images.

Herget suggested that the same star be put at different positions on the plate to estimate such an effect.

EDMONDSON noted that atmospheric refraction would affect all stars on the same plate nearly equally, and proposed taking plates in different altitudes and with different angles of the grating images relative to the vertical.

STOy made the point that it is frequently necessary to compromise quality slightly for the sake of quantity. He asked for opinions as to the range of magnitude that might be tolerated.

BROUWER said that for the Yale zone program the magnitude difference between central image and first-order spectrum was 3.5 magnitudes.

STOy inquired whether corrections for magnitude and color were introduced into the plate solutions at Yale, to which Miss BARNEY replied that they were.

Brouwer concluded the discussion by saying that gratings were important in considering future work and that a study should be made of advantages and disadvantages.

VASILEVSKIS was then called on to present a summary of his paper. He said two of the principal problems of the Lick program were the selection of stars and the choice of time interval. Since extragalactic nebulae had no actual proper motion of a measurable amount, their apparent proper motions in a fundamental system of coordinates would be caused only by errors in the adopted value of precession and systematic errors in the fundamental catalogue.

SHANE remarked that he was particularly interested in the discussion of the orientation of the grating images. The Lick Observatory uses a vertical orientation and no trouble had been encountered as yet. 
VAsilevskis stated that the accuracy of the stellar proper motions had been tested and that no systematic difference existed between red and blue stars on Io $X$ Io inch plates, but perhaps they might show up on the $I 7 \times$ I 7 inch plates. However, no measuring machine was available at the Lick Observatory at the present time for these large plates. He stated that if a time interval of 25 years is used between the two epochs this will give yearly proper motions with an accuracy of ".oo4 (p.e.). He further summarized considerations relating to the choice of stars on the program as outlined in his paper.

Vyssotsky commented that the entire program was an excellent one since it avoided the difficulties introduced by precession and that many of the results of the program cannot now be foretold. He indicated that the main difficulty in deriving the elements of galactic rotation would be their separation from the terms of solar motion in the least-squares solution, since only a portion of the sky is to be covered by the Lick program.

VASILEVSKIS replied that the elements of galactic rotation can be separated from the terms of solar motion if they are derived not from the whole Lick material but from a limited number of fields properly selected as to their distribution.

HECKMANN asked how nebulae were to be selected so as to avoid systematic errors. VASILEVSKIS said that nebulae must have sharp nuclei on both plates as seen in a blink comparator. He also advocated the use of the same kind of plates and the same exposure time for second-epoch observations, regardless of plate improvements which may be made in the mean time. In this way systematic plate errors may be minimized.

VYSSOTSKY said systematic errors in meridian observations depend upon right ascension and declination and consequently are not the same in low and in high galactic latitudes.

SHANE explained that there is a wide range of choice of nebulae, since most plates have at least 600 galaxies and some even 3000 ; even near the galactic plane the galaxies are sometimes visible through windows in galactic obscuration. Such windows have been found at Mt. Wilson and the zone of avoidance is probably not as large as it was thought to be by HuBBLE; hence more galaxies will be seen near the galactic plane than was originally anticipated.

NASSAU noted that many by-products were to be expected from the investigation and suggested that the positions of galactic windows be published.
Heckmann asked if it were possible to relate the Lick program and that of the $\mathrm{AGK}_{3}$.

VASILEVSKIS agreed that it was. A list of the brighter stars that are measurable on the Lick plates would be made available for all those planning other programs such as the $\mathrm{AGK}_{3}$.

STRAND noted that use of filters would be impractical in view of loss in magnitude and that therefore a color error was unavoidable. Consequently it would seem desirable to take a number of test plates to determine the range of effective wave lengths as soon as possible, before possible changes of color sensitivity of the plates took place.

VASILEVSKIs stated that such investigations are planned on a limited scale.

HERGET asked if there is any reason to suppose that a field correction exists which would lead to error.

SHANE answered that the color correction was too far to the red in a pilot model of the Lick astrograph, and that attention was paid to color correction in the design of the final lens.

HERGET asked if the correction could be well determined, and SHANE said that it could.

AldEN asked how stable the objective is to changes in the orientation of the telescope and if the telescope would be left without further adjustments.

HECKMANN recommended the taking of exposures of some fields in two positions of objective (telescope east and west of pier) to determine this stability. He also remarked that it would be unnecessary to use meridian circle positions directly for referring the fundamental system to the distant galaxies. Photographic positions rigorously reduced to a fundamental system would yield the same results.

BROUWER said that he completely agreed with this last remark.

\section{SECOND SESSION}

Sept. 3, 2 :30 P.M. R. H. Stoy, presiding

Scotr summarized his paper on "The U. S. Naval Observatory Transit Circle Program."

Vyssotsky asked how the proper motions would be derived from the observations, to which ScotT explained that one would use the catalogue positions and add a reduction such as from the Appendix to the PGC, thence reduce to the GC or $\mathrm{FK}_{3}$ system. One would then read the slope of the plot of positions against time. 
Vyssotsky observed that such results would reflect the systematic errors of the GC, unless corrections were applied to the GC system.

McClenahan then gave an account of the meridian work at Ottawa. A new program consists of observations of the Backlund-Hough list of stars and will be based upon the $\mathrm{FK}_{3}$ stars instead of Eichelberger's stars. Most recently only stars needed for special programs such as the PZT (Photographic Zenith Tube) program were being observed and would be completed this year. The next program would be to observe all $\mathrm{FK}_{3}$ stars north of declination $-27^{\circ} 30^{\prime}$ with a number of supplemental stars, a total of 3000 stars.

BROUwER asked if there were any expectations of continuing to observe stars of the PZT to check the meridian observations, to which McClenahan said he was entirely willing to continue such observations.

STOY asked if these observations were intended to supplement those of the PZT or to check the meridian observations.

Clemence thought it essential that some stars in each PZT list continue to be observed with transit circles for only in this way can time stars be placed on the same system.

Markowitz qualified Clemence's remark slightly by stating that meridian observations of PZT stars were needed for longitude rather than for time; Spencer Jones pointed out that the $\mathrm{FK}_{3}$ system has considerable periodic errors, which will tend to be smoothed out by the PZT.

SPENCER JoNES gave an account of the meridian circle work at Greenwich and the plans for future work at Herstmonceux. The Cook transit circle will be remounted at Herstmonceux with collimators and transit circle to be mounted in the same building. Azimuth markers will be used to control observations on single nights. Fundamental observations will be carried out with the Cook transit circle. The transit circle from Melbourne will be used for differential observations (non-fundamental work).

The future program will include fundamental observations of sun, moon, planets and reference stars for AG zones. Otherwise it is generally undecided. The observations of the $A G$ reference stars should be considered an important part of this program, provided sufficiently faint magnitudes can be reached.

There followed a general discussion of the relative merits of single- and double-wire micrometers, WATTS noting that a double-wire microm- eter has been used satisfactorily at Washington since I9I I and that it is possible with it to observe 9th magnitude stars on the best nights, though a limit of 8.5 magnitude is preferable.

STOY stated that a single-wire micrometer has always been used at the Cape.

Spencer Jones then called attention to the usage of making contacts audible, the rhythm of the ticks being helpful to the observer.

ScotT pointed out that ticks are advantageous at higher declinations and described WATT's method of employing ticks 4 seconds apart to give greater control to the observer.

MCCLENAHAN announced that BeAls hopes to build a telescope of the Atrinson type at the Dominion Observatory.

In answer to a question about the Lick transitcircle program, JEFFERs stated that it had been inactive for 20 years.

STRAND reported StRömGREN had made progress in the use of photoelectric cells for recording of transits, and believed he hoped to employ such a method with the new instrument in Denmark.

Spencer Jones noted that this would give a control of the personal equation.

HARRIS suggested the use of a photographic plate in the manner of the zenith tube.

DANJON reiterated reservations expressed at the Rome meeting of the IAU concerning the use of photoelectric cells.

Scotr warned of the possibility of a magnitude equation resulting from the response characteristics of a cell.

Heckmann emphasized this possibility and concluded that screening would be necessary.

Brouwer, Heckmann, and Spencer Jones discussed both sides of the question whether fainter stars should be included in meridian observation programs, agreeing that a number of the fainter stars could be left out.

HECKMANN stated that faint stars should not be omitted solely on consideration of the cost of including them, however.

MCCLENAHAN reported that a variety of colors of field illumination were being used to reach the fainter stars on the Dominion program.

STOY stated that red illumination was used at the Cape.

STOY launched a discussion concerning the necessity of meridian observations, pointing out their inefficiency in relation to photographic methods. 
Clemence noted that meridian observations were necessary for the determination of zero points of right ascension and declination.

SHANE asked whether we could expect an eventual completion of the meridian circle programs, noting that they were not necessary for the determination of proper motions as these could be determined from photographic work.

WATTS explained that meridian observations are used to establish a fundamental system and that to abandon transit observations would lead to deterioration of the fundamental systems.

MARKOWITZ raised the question whether the transit circle programs contain the minimum number of stars necessary to achieve their aims.

SHANE asked concerning the status of BROUWER's determination of the precession with the Loomis telescope.

BROUWER answered that a series of plates from which the location of the north celestial pole among the stars is to be determined has been taken since the end of $\mathbf{1 9 4 5}$, and is being continued. All but the plates taken during the last twelve months have been measured.

Clemence thought it would be agreed that transit circle programs cannot at the present time be dispensed with. He returned to the question of a minimum observing list.

HECKMANN recommended I5 stars per 25 square degrees and Miss BARNEy suggested 50 stars per Ioo square degrees. SToy noted that this would imply approximately 20,000 stars over the entire sky.

MARKowITZ asked whether it would be feasible to reduce the number of reference stars if fields were photographed in rapid succession using the plate constants from one field for the succeeding fields.

BROUWER thought it was not.

HERGET advocated an attempt to try the photographic method over a hemisphere, independent of meridian observations.

Spencer Jones, Schilt, and Vyssotsky expressed serious doubts as to the outcome of such a project.

Strand, Markowitz, and Stoy pointed out the great difficulties that would be introduced by differential refraction.

VAN BIESBROECK expressed himself as having long favored HECKMANN's procedure of recomputing positions from the Carte du Ciel after incorporating the $\mathrm{AGK}_{2}$ and $\mathrm{AGK}_{3}$ measures.
Spencer Jones pointed out that the only control of systematic errors was by observations over large arcs, and that whereas this is possible with meridian circles, it is not possible by photographic methods.

Brouwer turned the discussion to consideration of the ZVEREv list and read the following letter from ZvEREV pertaining to it:

"I. Reference is made to resolutions adopted by Commission 8 of the IAU at the Rome meeting. These call attention to the fact that Cape Observatory is the only one which makes meridian circle observations in the southern hemisphere. The commission further deems very important the installation in the southern hemisphere of an instrument similar to the Lick 20-inch.

" 2 . It is recommended that several observatories take part in the formation of a new catalogue of faint stars adopted in the USSR; especially important is the participation of southern observatories.

"The catalogue proposed in the USSR contains 20,000 stars from 7.5 to 9.2 vis. mag.; of these $93 \mathrm{I}$ are to be observed fundamentally, with 645 stars north of $-30^{\circ}$ and the balance south of that.

"The program also will include 300 areas with extragalactic nebulae (I2-I4 pg. mag.) and a selected list of minor planets. The meridian circle observations will start in $\mathbf{r} 955$.

"Please let me know whether your observatory has the facilities to undertake parts or all of this work. In case of a positive answer, we shall send you all the necessary detailed information.

"There are 7 observatories in the southern hemisphere which could join the program (Adelaide, Cape, Cordoba, LaPlata, Perth, Santiago, Sydney)."

VASILEVSKIS said that the Zverev stars are mostly too faint for the Lick program and contain too many red stars. Moreover, it is difficult to make direct comparisons between the extragalactic nebulae and the ZvEREv stars on the Lick plates. He also pointed out that it was advisable to decide now which program to adopt.

Scotr indicated that many of ZverEv's stars are too faint to be observed in Washington.

Vyssotsky stated that the Russian list was first made up in the I930's with a somewhat different plan in view, and did not see why it should 
be adopted if it was not satisfactory. The Russian list was originally confined to red giants because: I. they would be bright enough for meridian circles and yet faint enough on photographic plates for use as reference stars; and 2, they would have small motions and would be fairly evenly distributed over the sky so that the precession constants might be determined with higher accuracy.

\section{THIRD SESSION}

Sept. 4, 9:30 A.M. H. L. Alden, presiding

Vyssotsky, after summarizing his paper, emphasized that one must consider what can and cannot be achieved in reducing relative motions to absolute. If, as with the Cepheids, only relatively few objects with very small motions are available, then the cosmical errors must be reduced in every possible way; this would probably include the use of many faint reference stars of early spectral type. Observational errors must also be very small. Consequently, the entire project becomes exceedingly difficult to carry through successfully. In the case of long-period variables the situation is much more favorable since they are more numerous and their motions are larger.

HARRIS inquired if there were enough AG stars to be used as reference stars in the field of a McCormick plate. Vyssotsky replied that in an average field there were not enough and that they would introduce magnitude errors since they are usually too bright.

HECKMANN added that the difficulty referred to by Vyssotsky may be overcome by his proposal to proceed by intermediate steps from the AG positions to those of the Carte du Ciel.

SCHILT, in summarizing his paper, pointed out that the publication of the new parallax catalogue, which contains all the individual determinations of trigonometric parallaxes, has made his investigation possible. He pointed out that in reducing relative parallaxes to absolute the use of the present precepts frequently leads to negative corrections instead of positive. It is generally assumed that these so-called systematic errors will cancel out. However, taking the sky as a whole, and allowing for the parallaxes of the comparison stars, these corrections are negative with the result that the relative parallaxes appear to be too large. At the I948 IAU meeting at Zurich the question of reduction of relative paral- laxes to absolute was discussed and his viewpoint was accepted that the reductions are perhaps larger than appears from the mean parallaxes. This would imply that the systematic errors of the parallaxes, instead of being of the order of -".003 to - ".o05, would be as large as -".o08. The question whether this error depends upon the choice of comparison stars would be answered if it could be ascertained that two parallax determinations agree better when two stations used the same comparison stars. However, it is usually difficult to find out whether two stations used the same comparison stars, and the second best choice is to compare parallaxes measured relative to comparison stars that have nearly the same magnitude. The most noticeable systematic differences are between Allegheny and Yale, and especially for the very early Yale parallaxes.

BRouwer inquired which station, Yale or Allegheny, used brighter comparison stars when the difference of mean magnitude exceeded 0.3 .

Schilt replied that this would vary from star to star. He next discussed the comparison of trigonometric parallaxes with spectroscopic parallaxes.

Morgan pointed out the possibility of a systematic variation of stellar parallaxes with galactic latitude.

Schilt explained that such a difference was to be looked for in a systematic variation of the comparison stars with galactic latitude. $\mathrm{He}$ and MoRGAN agreed that it would be extremely valuable to undertake to get spectroscopic parallaxes of comparison stars on a large scale.

ALDEN then suggested that there might be a systematic difference with galactic latitude inasmuch as the comparison stars would be more often identical in high galactic latitudes, owing to the limited number to choose from, than in low latitudes.

MORGAN called attention to the fact that no large difference in distance is needed to give an effect of the size required.

NASSAU and Schilt agreed on the need for data concerning the spectra of comparison stars especially those with negative parallaxes or in regions of negative parallaxes.

STRAND described attempts made in recent years to obtain from Vyssotsky's program the spectra of comparison stars for Yerkes parallaxes. He noted that, since the scale of the Yerkes plates is large, the spectra of the comparison stars overlap in many cases with nearby stars on the objective prism plates. The same is true for plates taken with other long-focus telescopes. 
HARRIS presented his paper. He mentioned that his investigation was a preliminary one and dated back to the time of the IAU report, according to which Miss Jenkins was to compile a new general catalogue of trigonometric parallaxes, and that in the interest of economy no new investigation be made of systematic errors and the internal probable errors of the individual series, but that the Schlesinger precepts would be adopted. He wished to see if improvements could be made. He had checked his results against Miss JENKINs' in the new general catalogue and found that they agreed well. He continued with his paper, mentioning the correlation between the Allegheny and McCormick parallaxes. He concluded by saying that a similar correlation was not found between the Yale and Allegheny series.

WAGMAN questioned whether the same probable error of unit weight could be used for one station throughout since some series had considerably higher probable errors than others.

STRAND remarked that the procedure of adopting an average probable error of unit weight for the plates taken with one particular telescope is the best for obtaining a realistic value of the probable error of a parallax determined in the early series when a parallax was often based upon 8 to ro plates.

Vyssotsky, Clemence, Schilt and Harris expressed the common opinion in the subsequent discussion that it was undesirable to add observations to a series if the discordance with other values was large and that it was not defensible to reject highly discordant observations.

STRAND explained that his paper was intended to give a general summary of our present knowledge in the field of double star astronomy. The programs at Johannesburg and Bloemfontein had essentially completed the surveys for binaries down to the ninth magnitude. Lack of accurate magnitudes and spectra made the material unsuitable for statistical purposes and such studies should be confined to a lower magnitude limit, perhaps $6.5 \mathrm{I}$ on the Harvard scale as in KuIPER's survey. In the routine observations of relative positions of components in binary systems there had been a steady decline over many years. To compensate for this decline it was of utmost importance that emphasis be placed upon the photographic method leaving the pairs with separations less than $2^{\prime \prime}$ for the visual observer. This would require that fairly large instruments be made available for the visual observers. He also emphasized that further progress in the field depended upon additional accurate data on proper motions, parallaxes, mass ratios, magnitudes, spectral types and radial velocities. He thought that one of the reasons why so few people entered this field was the difficulty of selecting an observing program based upon the present general catalogues of double stars which are now more than 25 years out of date.

MARKOwITZ remarked that he felt that a worthwhile program could still be selected from Aitken's catalogue.

STRAND pointed out that the lists of observations of recent years do not always show evidence of careful planning which must in part be ascribed to the lack of up-to-date references.

MORGAN remarked that there is room for observations by all persons interested in double stars but if effort is to have weight, the programs should be made as determinate as possible.

JEFFERS volunteered the assistance of the Lick Observatory in making suggestions from their card catalogue in regard to observing programs.

VAN BIESBROECK noted that close pairs, which generally show the most rapid motion, are observable only with the largest instruments and therefore recommended that some time in the observing schedules of all such instruments be devoted to double stars. He specifically remarked upon the excellence of the work that can be done with large reflectors, such as the 82 -inch at McDonald Observatory, and hoped that a number of such instruments would be used for double star observations.

Spencer Jones remarked that the Greenwich Observatory will continue to observe double stars with the 28 -inch refractor.

STOY asked if there were to be a new double star catalogue.

Jeffers answered that Aitken's card catalogue had become the property of the Lick Observatory and that it has been supplemented with subsequent observations. At the present time the catalogue is essentially up to date. The Observatory frequently receives requests for data on one or several double star systems. Although it has often taken a week or more to copy the observations off the cards, arrange them in chronological order and type them, every effort has been made to fill the requests. At the present time the observations back to I927.0 are being transferred to IBM cards. The advantage of the 
punched-card system is that copies can be obtained with a minimum amount of work and that it will greatly facilitate the work of producing a new general catalogue. By agreement with VAN DEN BOS, who maintains the general catalogue for the southern sky, the Lick Observatory now limits its catalogues to north of $-20^{\circ}$ declination, instead of $-30^{\circ}$ as in the past, since the southern catalogue goes from $-19^{\circ}$ to the south pole. In connection with the preparation of a new general catalogue a circular letter had been written to all members of Commission 26 of the IAU. Questions were asked to serve as a guide in the preparation of the catalogue. The work is still in the planning stage and in the meantime reasonable requests for double star information will be filled by the Observatory. The Observatory also welcomes the receipt of new data for inclusion in its file.

ALDEN inquired if data were desired on stars with common proper motion and dwarf $M$ stars of wide separations.

JEFFERS confirmed that such data are desired.

STRAND asked if the Lick Observatory would prefer to receive new data already punched on cards.

JEFFERS said that this would be preferable, and offered to exhibit such punched cards and to explain the punch code.

\section{FOURTH SESSION}

\section{Sept. 4, 2 :30 P.M. A. Danjon, presiding}

The Chairman remarked that the discussions he had heard in the previous sessions were of great importance to the future of astrometry and that he would like to come back to the very important question whether photocells were appropriate for recording of transits. He said that this was not the opinion of Professor LALLEMAND and further stated that cells are appropriate for measuring constant brightnesses because they work well as integrators. If the time constant of the galvanometer is 2 or 3 seconds, the record is smoothed by integration. The transit problem is, however, quite different because when the cell is used for determining sudden variations of light, it works as a "derivator." It is well known that a good integrator may not be a good derivator.

Fluctuations of the photoelectric current are unavoidable. When the cell is free of secondary emission (which is the case with photomultipliers made by DR. LALLEMAND), the amplitude of fluctuations is proportional to the square root of the intensity. If a coarse grating is placed in the focal plane, the light variations resulting from the successive occultations of the star will be obliterated; first by the light of the bright sky, which is large compared with the light of the star; secondly, by the fluctuations.

Danjon then mentioned that HECKMANN had pointed out in a previous session that the magnitude equation could be eliminated by cutting down the brightness of bright stars. Since it is impossible to weaken the light of the star without weakening the light of the background sky, the magnitude equation is reintroduced in that way.

Better results could perhaps be obtained by using the photoelectric current for driving a traveling micrometer, which carries a knife with the edge polished as a mirror. The direct light of the star falls upon one cell while the reflected light falls upon a second cell. It would be easy to arrange things. so that the traveling knife edge is maintained on the star image. But in that case again, the magnitude equation cannot be completely avoided.

DANJON wished to emphasize the advantages of visual observations. He realized that many astronomers are getting away from that method nowadays because of the difficulty of securing the capable and experienced observers needed for that type of work, and he questioned if any automatic instruments can safely be put into the hands of incapable observers. Visual observations are at the present time the only way to utilize completely the resolving power of the telescope. This advantage vanishes with bad seeing, but under good conditions a high degree of accuracy can be obtained.

He further stated that the diameter of the diffraction pattern can usually be assumed to be of the order of 15 microns. Since the diameter of spider wires found in Paris is 8 to Io microns, the main part of the diffraction pattern is hidden by the wire, and the setting is determined by the external parts of the image, which are mostly disturbed by aberration. In his opinion, the best visual micrometer is the double-image micrometer. Unfortunately, it is not usable with a meridian circle.

The impersonal prismatic astrolabe which has been in use a few months in Paris for time and latitude determinations is equipped with a special double-image micrometer. When making the lens 
for this instrument, he assumed a probable error equal to 5 per cent of the diameter of the diffraction pattern, or 0 ". 4 . The first observation showed that this amount had to be divided by 2 . When the seeing is inferior, the mean quadratic error of a star observation is 3 to 4 per cent of the image diameter, and only 2.5 per cent when seeing is good. No photographic observation can reach such precision.

For further details about his instrument DANJON referred to his abstract in Comptes Rendus des Séances de L'Académie des Sciences 237, 28I, I953, and mentioned that the French stations will be equipped with such astrolabes, probably of larger size, during the International Geophysical Year I957-58.

In regard to the new prismatic transit instrument, described in the paper submitted to the Conference, he had nothing to add because it was set up at the Paris Observatory later than expected and its adjustment was not complete. Since July was rainy in Paris, he had only once had the opportunity to look at stars through the new instrument. It was sufficient to check, as he had done already with a smaller experimental instrument, the reliability of upper and lower transits of circumpolar stars. The residuals found for the circumpolar stars were as follows, in milliseconds :

\begin{tabular}{|c|c|}
\hline sits & Upper transits \\
\hline $\begin{array}{ll}\text { I H Cep } & + \text { I } 4 \\
\text { Groomb. I374 } & -7\end{array}$ & $\begin{array}{l}\text { Groomb. } 3212-27 \\
73 \text { Dra }\end{array}$ \\
\hline
\end{tabular}

Following Danjon's remarks, discussion centered upon the possible use of photoelectric cells to record transits.

SPENCER Jones expressed the hope that photoelectric observations might avoid some of the difficulties of conventional instruments. He asserted that he did not consider LALLEMAND's results conclusive and was conducting his own experiments on the subject.

Vyssotsky inquired if the light of the sky were important, to which DANJON answered that it was, since the sky background and Schottky effect would tend to mask a signal.

Markowitz outlined the substance of his paper adding several slides and a few explanatory remarks. He was hopeful that some ro to 20 observatories would be able to participate in the program of determining lunar positions photographically and that preparations would be completed in time for the observance of the third Geophysical Year, 1957-58. These observations would also give important data in regard to changes in the earth's rate of rotation. He cited recent changes over the period Dec. I952 to Aug. I953 as obtained from the photographic zenith tube observations compared with crystal clocks and mentioned that the lunar observations would throw further light on these changes. Other advantages of the photographic method were not only higher accuracy but the fact that a large portion of the moon's orbit can be observed. By combining northern and southern hemisphere observations it would be possible to observe over 22 days of each lunation.

BROUWER remarked that crystal clocks, corrected over longer intervals of time with the aid of precise photographic observations of the moon's position, should be capable of giving a much more detailed record of the changes in the rate of rotation of the earth than can be obtained from occultations and meridian circle observations of the moon. He hoped that the U.S. Naval Observatory would use the old methods of observing the moon as well as the new and thought that an overlap of ro years was advisable.

NAssau asked if a difference in contrast between the plate and the moon image would affect the measures of the moon's limb.

MARKOWITZ stated that though different persons determine positions of limb differently, the measurements lead to the same center of the moon.

SPENCER Jones noted that meridian circles give large systematic errors in lunar observations and expressed the opinion that Markowitz' photographic method was considerably less vulnerable. He wished to know how the parallelism of the filters is tested, to which MARKowITz replied that it is done by coincidence of reflected images of distant objects. Moreover, provisions had been made for turning the camera $180^{\circ}$. By reversing the camera, the errors in paralleling will cancel out and timing will be accurate.

NASSAU inquired if non-uniformity of density over the moon's limb would cause systematic errors, to which MARKOwITZ replied that it would lead only to accidental errors for the night. He added that the photographic image of the moon appears prolate by 0 ". 8 due to a high mountain range on the south limb.

STRAND wished to know if the starlight passed through the same filter thickness as the moonlight. MARKowitz answered that it did.

Spencer Jones concluded the discussion by stating that the method seemed to be one of very 
great promise, superior to the traditional methods of meridian observations and occultations, though the latter should be continued for a time as a check on the new method.

BAKER began the discussion of improvements of technique and materials in the field of optics since the last war. He noted that electronic computers had enormously reduced the computational work in lens design and had been used extensively at Harvard. He cited as an example that an II-element apochromatic system could be fully calculated on an electronic machine in I 2 minutes, in contrast to the 6 months it would require if ordinary desk calculators were used. He mentioned that the situation of getting optical material is near perfection and that it is now possible to get objective disks of interferometer quality up to 40 inches in diameter. The striation problem has been largely overcome at Corning by the use of a platinum blender. Moreover, indices of refraction can be controlled to within 2 or 3 units in the 5 th decimal place. Problems of mounting and adjusting objectives may be satisfactorily solved by improved engineering. In a similar manner tube flexures and vibrations may be greatly reduced. The diffraction image becomes asymmetric on account of distortion of the spherical wave front. Work is now in progress to catalogue the sensitivity of a great variety of lens systems to deflections of tilt adjustment, flexures and temperature, so that it should soon be possible to make a ready selection of the optical system best suited to any given requirements. BAKER then described the design of the catadioptric refractor, which seemed to offer promise of application to astrometric problems.

Heckmann inquired if a Schmidt telescope could be used astrometrically.

BAKER replied that large Schmidts lack adequate accuracy in figuring and that the images are therefore asymmetrical, adding that even so they are of the order of a hundred times better than those of a paraboloid.

SHANE noted that images formed by a Schmidt telescope on different parts of the plate come from different parts of the mirror.

HERGET asked if flexure of the correcting plate would introduce error and BAKER thought it would not be serious.

Vasilevskis informed the Conference that the possibility of using a Schmidt astrometrically could be investigated at Palomar, but cautioned that not much is to be expected from the use of Schmidt telescopes for precise astrometry.
HARRIS asked what set of stars could be used to test a Schmidt, to which Heckmann answered that any set of stars could be used if several meridian circles were employed as a check.

STRAND observed that there might possibly be different plate constants over various parts of the field of a Schmidt.

VASILEVSKIS pointed out that if this were the case only a small field could be used, and there would be no point in using a Schmidt telescope at all for astrometry.

ECKERT then presented a summary of the paper by himself and Miss R. B. Jones on automatic measurement of photographic star positions. A series of experimental measurements gave the probable error 0.56 micron for a single automatic setting on a star image.

Clemence wished to know how this setting error compared with the error inherent in the photographic plate.

MisS BARNEY stated that she would estimate that the latter error was of the order of 0.5 micron. (This is based on results obtained by T. G. Menlin that were published in A. J. 44, I69, I935.)

In the discussion that followed the consensus of opinion was that further improvement in the accuracy of measurement was not important at present in view of the fact that the photographic process introduces a probable error as large as 0.6 micron in the position of an image on a plate.

STRAND remarked that the image error in double star measures with refractors of long focal lengths were of the order of $\mathbf{I} .5$ to 2 microns, as shown by variation in the position of the grating images. If the automatic measuring machine would duplicate the accuracy obtained for the Yale plates for this type of measurements then it was highly accurate.

Heckmann pointed out that the measuring error in the AGK2 program was one micron for the mean of two positions on one plate, and that several plates had errors twice as large.

Duke wished to know what the errors were for the visual measures, to which Eckert replied that for a single plate the Yale residuals for the Washington comparison stars gave the probable error o". I79, while the corresponding probable error for the residuals of the automatic measurements is 0 ". I89. In both cases this probable error contains the uncertainty of the meridian circle position as well as the error of measurement and the error inherent in the photographic plate. 
The discussion turned next to the work of revising the $\mathrm{FK}_{3}$. Heckmann related that this work is principally being carried out in Heidelberg by KOPFF and his associates, and is intended to be completed within the next five years. The time service carried out at Hamburg is used for the purpose of checking systematic errors in the $\mathrm{FK}_{3}$ system and the errors of the crystal clocks. No attempt is made to determine the non-uniform rotation of the earth. The program uses the quartz clocks at Hamburg, Heidelberg and those of the German North West Radio Society, and both observatories are observing with broken transits during the whole night. For certain ranges in right ascension the corrections to the FK 3 system so determined appear to be similar to the differences between the $\mathrm{N}_{3} \mathrm{O}$ and $\mathrm{FK}_{3}$ systems.

SPENCER Jones asserted that approximately $\frac{1}{3}$ of the variation in the rate of the earth's rotation is due to errors in the $\mathrm{FK}_{3}$, if $\mathrm{N}_{3} 0$ may be taken to be correct.

Clemence added that in making time determinations with the photographic zenith tube it is possible to conform to the $\mathrm{FK}_{3}$ in a general way only. Since it is important that the right ascensions be referred to the equinox of the $\mathrm{FK}_{3}$, it is desirable that the PZT stars be kept under observation with the transit circles. Only in this way can the equinox of the $\mathrm{FK}_{3}$ be preserved.

SPENCER JONES pointed out that it was important to have the revision of the $\mathrm{FK}_{3}$ completed prior to the meeting of the Congress on International Longitude Determinations in 1957 or I958.

\section{FIFTH SESSION}

\section{Sept. 5, 9:30 A.M. O. Heckmann, presiding}

MORGAN began with a summary of the recent meeting at Groningen on galactic structure. $\mathrm{He}$ wished to emphasize that what he was reporting were his own conclusions based upon the discussions at that conference. He was impressed by the change in point of view that has recently taken place with regard to observational methods of stellar astronomy. At no time were suggestions made to embark on new large-scale investigations which would make use of the statistical methods of the past, such as general star counts. The present trend is to more accurate detailed investigations, in recognition of the fact that the statistical results are frequently vitiated by system- atic errors. One of the specific changes in observational method has been the replacement of photographic photometry by photoelectric methods. All the new methods and attitudes call for a close scrutiny of systematic errors.

The portions of the Groningen Conference which were of special interest to astrometry included: I. A reobservation of the AG zones; 2. A proposal to observe two new classes of objects divided into arm stars and disc stars. The disc stars show a strong concentration in galactic longitude in the direction of the galactic center and a smooth featureless decrease with distance from the galactic center; they include the RR Lyrae stars, novae and planetaries, while the arm stars include blue supergiants and supergiants of early $M$ type. A most promising group of the disc stars is an unpublished list of the brightest stars of classes $M_{5}$ and later in the galactic belt, in northern longitudes. At Groningen there was a request for a list of such stars so that they might be reobserved for determination of their proper motions. This is a difficult problem since the expected motions are small.

He further stated that in regard to the B stars there is a great need for a definitive calibration of their spectroscopic parallaxes. At the present time the zero point of this calibration depends upon main sequence $B_{3}$ to $B_{5}$ stars; while the scale has been set from clusters with sufficient range in absolute magnitude.

It was formerly thought that the task of discussing the proper motions of the B stars could be considered as simple, but with the discovery of expanding associations, the problem has become complicated, because the motion of expansion may overshadow the reflex of the solar motion. Since there appears a sudden change in the distribution near $\mathrm{BI}$, the motions of $\mathrm{B}_{3}$ and $B_{5}$ main sequence stars should be investigated because they can be considered to have a more nearly random distribution.

It would also be of special interest to observe the more distant members of the $\mathrm{O}$ associations to a distance limit of approximately Iooo parsecs from the sun for the determination of possible large space motions.

MoRgAN continued with a description of astrometric problems of galactic structure related to the O-type stars (cf. p. 86).

HeCKMANn remarked that Morgan had already outlined that there is a need for accurate proper motions of blue stars and red giants, and 
that the discussion should be centered upon which methods should be used and what accuracy could be obtained.

Clemence wished to know the distance of the red and blue stars referred to by MorGaN.

Morgan cited the $\zeta$ Persei association as the nearest at about 300 to 400 parsecs, the Orion group at about 500 parsecs, and the group in Cepheus at about 700 parsecs. All have small proper motions.

Clemence remarked that he had in mind that there would be no effect of parallax in these proper motions.

HECKMANN recommended that the determination of the proper motions of these stars should be undertaken only by an experienced astrometrist who could estimate reliably the precision required of a second-epoch measure in order to obtain a sufficiently low mean error for such small proper motions.

Clemence suggested that H. R. Morgan of the Naval Observatory might be willing and able to do a moderate amount of such work. He could decide what observations with meridian circles were needed in the next year or two. Such stars would be added to the current observing list at Washington.

HECKMANn added that the Bergedorf Observatory would share in such a program.

MoRGAN pointed out that if we could determine the approximate direction of motion of these objects even to within only $10^{\circ}$ or $15^{\circ}$, this would be of great importance in helping to determine their space origins, and from this the age of the group of which they are a part.

BROUWER wished to know if it were possible to improve the proper motion determinations by including these stars on the program of a longfocus telescope. It was HeckmanN's opinion that high accuracy could be achieved in such cases by the use of a rotating sector and an interval of ten years.

MORGAN pointed out the need of absolute rather than relative proper motions, and asked what accuracy could be had for them.

Vyssotsky remarked that the McCormick Observatory might soon be able to give a tentative answer to this question since Mitchell had put many $B$ stars on the program.

MoRGAN objected that this program makes use of differential methods.

Vyssotsky recommended that it be tried on isolated $B$ stars. It should give good results if distant reference stars are used, such as A and F stars with small dispersions in velocity. From this one might determine whether it is worth while to institute a new program with a longfocus instrument.

Alden inquired if only a few proper motions would be worth-while. MorGan replied that they would be valuable for individual objects of special interest.

Herget wished to know if astrographic plates could be used provided the interval were sufficiently long. HECKMANN thought not, since the magnitude equation would be too serious.

Morgan added that very few red giants of the long-period variable type would appear on astrographic plates because they are at maximum for a short time only and because they are so faint in the blue.

NAssau stated that the Warner and Swasey Observatory is observing a number of red stars down to 8.2 infrared magnitude, selected as the brightest of a list of about 5000. The selected stars have a limiting photographic magnitude of I2.5, and Io per cent are long-period variables. He suggested that an expedient method of obtaining the proper motions of these long-period variables would be to have someone examine the numerous astrographic plates and if possible identify the variables on them. If some of these stars are found in the various zones of the Carte $\mathrm{du}$ Ciel, it might be possible to persuade Commission 23 of the IAU to have these zones reobserved for second-epoch plates.

ALDEN announced that the relative proper motions of more than 200 long-period variables distributed over the sky have been measured on his current program. The probable errors as indicated by VyssoTsky's paper are of the order of ".o03.

VysSOTSKY added that these proper motions could easily be reduced to absolute motions because the spectra of the reference stars are available. He wished to know if information on the RV Tauri stars is desired and Morgan asserted that it would be very welcome.

Heckmann remarked that the stars on NAsSAU's list for which proper motions may be found from the Carte du Ciel may possibly overlap with ALDEN's list of long-period variables.

HARRIS stated that the Lowell Observatory collection contains survey plates for epoch I930 coinciding with the epoch of $\mathrm{AGK}_{2}$ and covers the sky to $-30^{\circ}$ declination to the 17 th magni- 
tude. There are at least two perfect plates for each field, both early and late. The scale is about $120^{\prime \prime} / \mathrm{mm}$. The survey will be repeated in 1960 and will possibly give proper motions to "oor.

BROUWER commented that since no grating had been used in this survey it would be difficult to relate these proper motions to those of the AG stars.

Heckmann suggested that the first-epoch plates should be obtained with a long-focus refractor for the stars of NAssau's list, which are too faint to be on the Carte du Ciel.

MORGAN stated that if the reduction from relative to absolute motion is not too difficult, longfocus refractors could be used.

Vyssotsky thought the problem should be considered star by star.

STRAND suggested that a survey of spectra of comparison stars be made to supplement this work.

Vyssotsky stated that the McCormick Observatory has objective prism plates down to $-15^{\circ}$.

SToy asked if some of this work could be done with the ADH telescope, to which Vyssotsky agreed, but BAKER pointed out that the future operation of this telescope is quite uncertain.

HECKMANN explained that in conversations with BoK he had learned that the ADH telescope would be made available to investigators who will defray their own expenses.

HECKMANN thought it was desirable to have the astrophysicists supply a list of objects for which they would be particularly interested in having astrometric data and cited as an example secular parallaxes of Cepheids.

Alden mentioned that this question was brought up at the last IAU meeting in Commission 24, Stellar Parallaxes and Proper Motions. No definition of objects of astrophysical interest was made, but it was left up to the chairman to prepare a list of Ioo stars in this category. He intended to circularize members of the IAU interested primarily in astrophysics in regard to the content of the list. He emphasized that the astrometric people would tend to load the list with problems of dynamical interest, whereas the astrophysicists would want special groups observed to establish zero point and set up criteria.

NAsSAU stated that lists would gladly be supplied by the Groningen Conference.

HECKMANN stressed particularly both the astrometric and astrophysical interest in star clusters.
Herget then directed the discussion to the rather more special topic of the observation of minor planets. He acknowledged the value of KUIPER's survey of the ecliptic for the purpose of a statistical investigation of the minor planets. This has proved of great help in eliminating misidentifications. He therefore proposed that such a survey be carried out every decade or so and that: $\mathbf{I}$. some particular institution accept responsibility for taking the survey plates; 2 . the plates be blinked to detect the minor planets ; 3 . the positions of the planets be measured (possibly on an automatic measuring machine); 4 . the measures be reduced preferably by means of an automatic calculating machine according to the procedures now in use at Cincinnati. Such a plan promises to eliminate much of present minor planet observing, because most of the present observations are of bright objects and therefore of very limited value.

STRAND noted that since it was not necessary to measure the plates with high precision, the Kapteyn method recently revived and improved by VAN BIESBROECK would save both time and labor, to which HERGET agreed.

\section{SIXTH SESSION}

Sept. 5, I :30 P.M. G. M. Clemence, presiding

During this session the Conference deliberated several resolutions drawn up by a committee with SPEncer Jones as Chairman. These resolutions are printed on p. IO4.

Clemence invited Spencer Jones to read the first resolution.

The discussion following the reading of the first resolution was opened by HERGET, who noted that little consideration had been given to the possibility of new and improved astrometric techniques, which might alter future programs. Specifically he inquired as to plans for the use of Atkinson's horizontal meridian circle.

Spencer Jones replied in general terms that it was desirable to make observations by as many methods as possible since this is the best means of controlling systematic errors.

Clemence wished to emphasize at this point that many fields had not been covered by the Conference and that questions of a general nature should be considered rather than specific details. In speaking for the resolution, he emphasized that an $\mathrm{AGK}_{3}$ would give a homogeneous system 
of proper motions of high systematic accuracy, and that the results would be important to a considerable number of people. He felt that if the resolution was adopted it might have a significant influence in determining the activities of a large group of astronomers during the next few years.

HERGET raised the question which list of stars should be observed. He suggested that ScotT's list be revised so that there be none fainter than magnitude 8.5 , that there be approximately 0.5 stars per square degree, and that no choice be made by spectra.

At this point it was remarked that the ZvEREv list contained many stars fainter than this limit and gave considerable weight to red stars, which are faint photographically.

It was noted too that the $\mathrm{AGK}_{3}$, whose limit was set at -2.5 declination should be extended to at least $-30^{\circ}$ in order to help the programs at Lick and Yale. It was also considered important that an extensive number of Anhaltsterne, since they had been observed before, and ZVEREV stars should be included so as to allow a good comparison between the two systems. The epoch should be close to I 958 and the list should include about 2 I, ooo stars. Suggested participants should include the U. S. Naval Observatory and the observatories at Bergedorf, Bordeaux, Copenhagen, Heidelberg, Herstmonceux, Munich, Ottawa, Paris, San Fernando and Uccle. At the close of the discussion the adoption of the resolution was moved, seconded and approved unanimously.

Following the reading of the second resolution Clemence explained that in the past the meridian circle program of the U.S. Naval Observatory was directed to the continuous observation of $\mathrm{FK}_{3}$ stars together with the sun, moon and planets, in order to preserve a fundamental system; to the observation of reference stars to be used in the construction of the Yale photographic catalogue; and finally to the observation of reference stars needed for the Lick Observatory survey. It is especially important that in taking up additional work, these three programs be continued in a satisfactory way. In another respect this resolution is important because until now the U. S. Naval Observatory has felt it had the responsibility of providing, in addition to these three programs, the proper motions of stars with good observational histories. He expressed his conviction that the U. S. Naval Observatory need no longer consider the requirement of proper motions which can be obtained with more homogeneity from photographic catalogues. The resolution, if adopted, might change the emphasis of the meridian work at the U. S. Naval Observatory in the direction of less regard for proper motions as such. The adoption of the resolution was moved, seconded and unanimously approved.

After the reading of the third resolution a brief discussion followed in regard to the possibility of moving the Lick telescope to the southern hemisphere when the program now in progress was completed. The general opinion was that this would not be feasible. The third resolution was then moved, seconded and approved unanimously.

After the reading of the fourth resolution informal offers of meridian circles that could be made available to southern observatories were made by Lick, Dearborn and Bonn.

HERGET noted that anyone engaging in a meridian-circle program in the southern hemisphere would find it necessary to modernize and modify donated instruments.

SToy called attention to the fact that at the most recent IAU meeting a similar resolution was adopted.

ALDEN felt that some astronomers might think that in passing these resolutions the members of the Conference were taking too much authority upon themselves.

SPENCER Jones thought this was not so, but that the resolutions would form a basis for consideration by the IAU. The IAU has the controlling authority on matters which affect all astronomers, such as the naming of variable stars and the use of fundamental star systems. Certainly any group of astronomers is perfectly free to express its opinion on any matter.

The adoption of the resolution was moved, seconded and approved, with one abstention and no contrary vote.

The fifth resolution was read, moved, seconded and unanimously approved.

A final discussion was initiated by Clemence, who asked what the optimum accuracy of a measuring engine should be in relation to the precision obtainable on a photographic plate. It was HECKMANN's opinion that there should be a proper relation between the precision of the measuring instrument and that of the photographic plate, the former being more precise than the latter. Since the error of a plate is now not 
less than one micron, the precision of a measuring engine should be somewhat less than I micron, but need not be as low as o.I micron.

ScHilt dissented, pointing out that if the accuracy of the measuring apparatus is not markedly greater than that of the plate, there is less reason to attempt a greater improvement in the plate accuracy.

SPEncer Jones offered as an example of improved plate accuracy the early parallax procedure at Greenwich, where three exposures were made with a displacement in declination. Subsequently two exposures were made, the plate being reversed between exposures. The resultant probable error from the two exposures was less than that from the three because of a reduction of the plate error, by eliminating the effects of shifts in the emulsion.

Clemence asked if it were not advisable to eliminate the largest error first, which in this case was the plate error.

ScHILT noted that many questions can be answered more readily if errors of measurement are much below errors from other sources.

Heckmann and Schilt agreed that there is no profit in great accuracy of measurement when applied to a diffuse object.
Markowitz wished to differentiate between the use of a machine at one observatory for one specific program and the use of several machines of the same type at different observatories in a coordinated program. In the second case small systematic errors will cause trouble. In order to have this error cancel out, should one specify whether the machine would be required to measure to an accuracy of one or two microns?

Spencer Jones remarked that as long as the systematic errors cancel out, any accuracy will do, up to a certain limit.

ALDEN cited the custom of using four decimal places in parallax work. After dropping the fourth decimal place, the same final results were obtained. Since the probable error of the plate was between I and 2 microns, the use of the fourth decimal place is only a waste of time and labor.

The session concluded with a vote of thanks to the National Science Foundation for making the conference possible by its support, to Northwestern University for housing the conference, to Dr. K. Aa. Strand for the arrangements, and to Dr. W. S. Krogdahl and MrS. E. VAN INWAGEN, who acted as recorders of the sessions. 